\title{
Field Synergy Principle For Natural Convective Rotating Fluid Flow Past a Vertical Cylinder
}

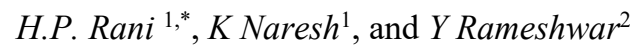 \\ ${ }^{1}$ Department of Mathematics, National Institute of Technology, Warangal-506004, India \\ 2 Department of Mathematics, University College of Engineering, Osmania University, Hyderabad-500007, India
}

\begin{abstract}
In the present research work, the effects of thermal and induced magnetic fields on the natural convective rotating fluid flow past the vertical cylinder are presented. The numerical solution of the unsteady field variables is obtained by solving the governing non-dimensional non-linear equations. The effects of the Prandtl, Taylor and Chandrasekhar numbers on the induced magnetic field, average momentum and heat transfer coefficients are presented graphically. The field synergy/ coordination principle is discussed to understand the enhancement of convective heat transfer. Increasing values of synergy co-insides with the increasing rate of heat transfer.
\end{abstract}

\section{INTRODUCTION}

The studies related to the natural convective flow past a vertical cylinder are significant due to the wide range of applications in the field of geothermal and geological formulations. Gregg [1] originally researched this study of heat transfer related to vertical cylinders. Globe [2] evaluated the time-independent flow of an electrically moving fluid lying between two lengthy circular cylinders in the annular space with the existence of a magnetic field. In addition, by using the Laplace transform method, Goldstein and Briggs [3] evaluated the unstable free convective flow past the circular cylinder and vertical flat plate. Under the impact of a radial magnetic field, Dube [4] paid attention to the laminar flow between the concentric rotating porous cylinders of an electrically conductive fluid. One of the latest developments in magnetohydrodynamics is the natural convective flow along the vertical cylinder. Heat transfer improvement plays a vital role in any thermal conductive experiment. It relies on the angle between the gradient of temperature and speed. Guo et al. [5] are initiating this phenomenon. This principle describes the heat transfer improvement and Guo et al. [6] details it. Guo [7] disclosed that the intensity of convective heat transfer depends on temperature gradient, velocity and fluid characteristics, along with the included angle between velocity and gradient temperature fields. In recent years, much interest is focused in this emerging area because of its wide range of applicability in oceanography and geothermal power generation, geophysics, astrophysics, agriculture, engineering, and technology.

It is interesting to observe the development of the flow over a vertical cylinder for the incompressible fluid flow, which is kept in a rotating fluid. The fluid that rotates induces the power of Coriolis. In this present work, an incompressible viscous fluid past a semiinfinite solid circular cylinder is considered. The fluid and the cylinder are kept in a constant horizontal magnetic field. The fluid rotates in the tangential direction with uniform angular velocity. The current research focuses on the numerical solution for the velocity, temperature variables and induced magnetic field that is acquired from the solution of the governing non-linear differential equations linked to the natural convective flow past a vertical cylinder.

\section{MATHEMATICAL FORMULATIONS}

We have considered the free convective viscous incompressible electrically conducting fluid flow past a semi-infinite vertical cylinder in the presence of an applied induced magnetic field is as shown in Fig. 1. Constant horizontal magnetic field of strength $B_{0}$ is assumed. The induced magnetic field produced by the motion of the fluid is considered. The radius of the cylinder is taken as $r_{0}$. The initial temperature of the cylinder is taken as $T_{\infty}^{\prime}$ and later it is increased and maintained at $T_{W}^{\prime}$. Since the considered rotating system is non-inertial, the Coriolis force will exist. Considering the uniform pressure in the entire fluid sector, the governing equations linked to the physical model are as follows under the Boussinesq approximation $[1,4,8]$ :

$$
\begin{aligned}
& \frac{\partial(R U)}{\partial X}+\frac{\partial(R V)}{\partial R}=0 \\
& \frac{\partial U}{\partial t}+U \frac{\partial U}{\partial X}+V \frac{\partial U}{\partial R}-\sqrt{T a} V=G r T+\frac{1}{R} \frac{\partial U}{\partial R}+\frac{\partial^{2} U}{\partial R^{2}}+Q \frac{\partial H}{\partial R} \\
& \frac{\partial V}{\partial t}+U \frac{\partial V}{\partial X}+V \frac{\partial V}{\partial R}+\sqrt{T a} U=\frac{1}{R} \frac{\partial V}{\partial R}+\frac{\partial^{2} V}{\partial R^{2}}
\end{aligned}
$$

\footnotetext{
* Corresponding author: hprani@nitw.ac.in
} 
$$
\begin{aligned}
& \frac{\partial H}{\partial t}+U \frac{\partial H}{\partial X}+V \frac{\partial H}{\partial R}=\frac{1}{P m}\left[\frac{1}{R} \frac{\partial H}{\partial R}+\frac{\partial^{2} H}{\partial R^{2}}\right]+\frac{\partial U}{\partial R} \\
& \frac{\partial T}{\partial t}+U \frac{\partial T}{\partial X}+V \frac{\partial T}{\partial R}=\frac{1}{P r}\left[\frac{1}{R} \frac{\partial T}{\partial R}+\frac{\partial^{2} T}{\partial R^{2}}\right]
\end{aligned}
$$

The initial $(t \leq 0)$ and boundary conditions are as follows

$$
\left.\begin{array}{c}
t \leq 0: U=0, V=0, T=0, H=0, \quad \forall X \text { and } R \\
t>0: U=0, V=0, T=0, H=0, \frac{\partial H}{\partial R}=0 \\
\text { at } X=0 \\
U=0, V=0, T=1, H=0, \frac{\partial H}{\partial R}=0 \text { at } R=1 \\
U \rightarrow 0, V \rightarrow 0, T \rightarrow 0, H \rightarrow 0 \text { as } R \rightarrow \infty
\end{array}\right\}
$$

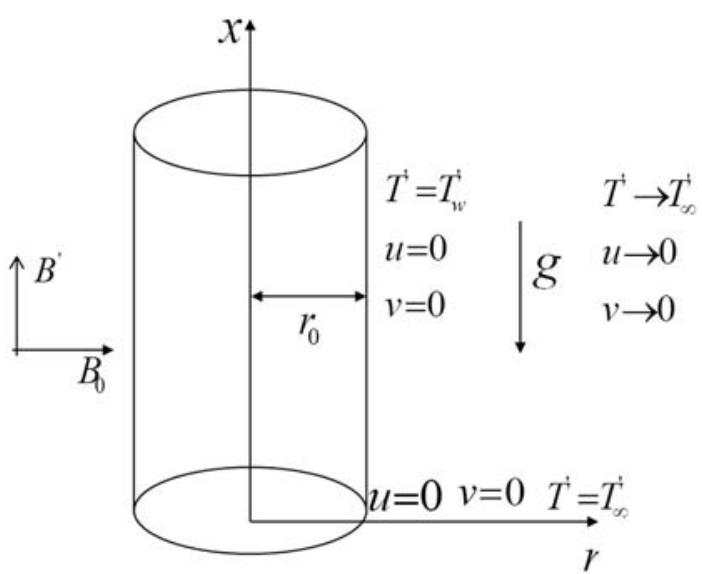

Fig. 1. Schematic of the investigated problem.

The employed non-dimensional variables are $t=\omega t^{\prime}, X=\frac{x}{r_{0}}, R=\frac{r}{r_{0}}, U=\frac{u r_{0}}{v}, V=\frac{v r_{0}}{v}, T=$ $\frac{T^{\prime}-T_{\infty}^{\prime}}{T_{W}^{\prime}-T_{\infty}^{\prime}}, H=\frac{B^{\prime}}{B_{0}} . \quad$ In the above equations, control parameters are defined as $T a=\frac{4 \omega^{2} r_{0}^{2}}{v^{2}}, G r=$ $\frac{g \beta\left(T_{w}^{\prime}-T_{\infty}^{\prime}\right) r_{0}^{3}}{v^{2}}, P m=\frac{v}{\eta}, \operatorname{Pr}=\frac{v}{\alpha}, Q=\frac{B_{0}^{2} r_{0}^{2}}{\mu_{e} \rho v \lambda}$. The notations have the usual meanings and explained in nomenclature.

The non-linear Equations (1)-(5) are unsteady and coupled equations. A numerical approach is used to solve these equations. Here, Crank-Nicholson scheme, which is an unconditionally stable finite difference method, is employed to get the solutions. The grid system of $100 \times 400,50 \times 200,200 \times 800$ and $100 \times 400$ is compared with respect to the velocity and temperature values. The grid system of $100 \times 400$ showed variability in the fifth decimal place compared to the outcomes of $200 \times 800$. The mesh size of $\mathrm{X}$ and $\mathrm{R}$ is therefore set at 0.02 and 0.01 , respectively.

\section{RESULTS AND DISCUSSION}

The effect of $T a$ and $Q$ on velocity, magnetic field, momentum and heat transport rates analyzed. In all cases $G r=5$ and $P m=0.1$ fixed. The variations of axial and radial velocity profiles are graphically shown. For varying $T a$ and $Q$ values, the effect of Nusselt number and skin-friction are discussed.

Figure 2 demonstrates the axial velocity $U$ and transverse velocity $V$ profiles against the radial coordinate of the cylinder for distinct values of rotating parameter $T a$ when $Q=0.8$ and $P r=0.71$. The velocity profiles begin with the value zero at the leading edge of the cylinder, reach their maximum and then decrease for all time values to zero along the radial coordinate. The velocity profiles reduce for raising $R$ and $T a$.

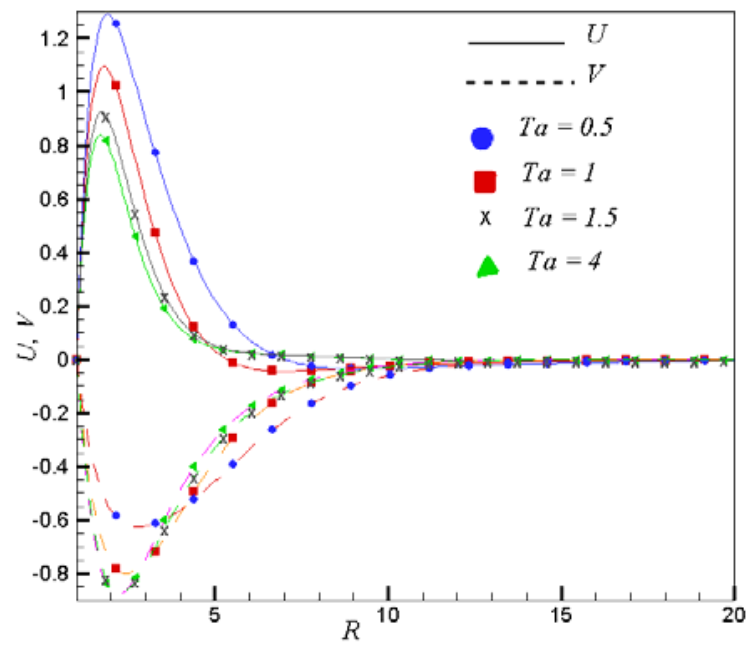

Fig. 2. Steady-state axial and transverse velocity profiles for different $T a$.

From figures 3 and 4 , it can be noted that when $Q=$ 0.8 and $P r=0.71$ the unsteady axial and transverse velocity profiles are smoothly reached the steady-state, due to the presence of Coriolis force. When $T a$ increases both the axial and radial velocity components have oscillations. Thus there is the existence of inertial oscillation that grows with time. When $T a$ is large, the viscous force can be large relative to the Coriolis force. When $T a$ increases velocity profiles decrease with respect to the time.

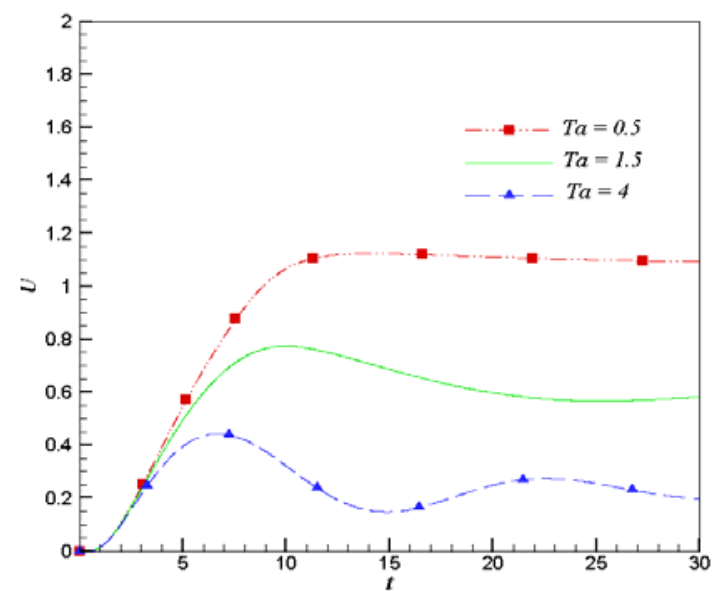

Fig. 3. Unsteady axial velocity profile for different $T a$ values. 


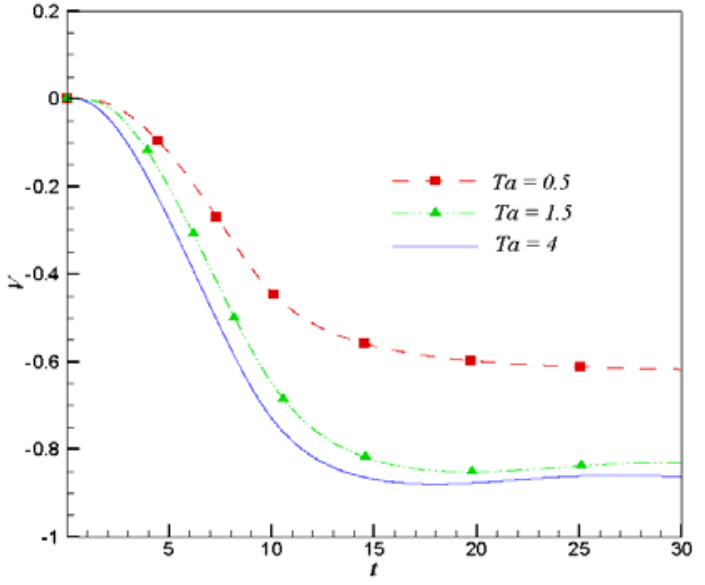

Fig. 4. Unsteady transverse velocity profile for different $T a$ values.

Figures 5 and 6 show that the thermal profiles at $G r=5$, $T a=4$ and $Q=0.8$ for different fluids i.e., different $\mathrm{Pr}$ values. As the $\operatorname{Pr}$ increases, the temperature decreases. From Fig. 6, it can be noted that the unsteady temperature profiles rise over time, reach a temporary maximum and then asymptotically reach a steady-state value.

The temperature rises initially for small time values, but with increasing time values the temperature values become constant. Also, with growing $\mathrm{Pr}$, the time to achieve the time-independent state is growing.

\subsection{Overall momentum and heat transport coefficients:}

For the heat transfer research, the wall shear stress is an significant parameter and is directly linked to the heat transfer coefficient. The non-dimensional average skinfriction coefficient or wall shear-stress is given by $\overline{C_{f}}=$ $\int_{0}^{1}\left(\frac{\partial U}{\partial R}\right)_{R=1} d X$. The effect of $T a$ on the simulated $\overline{C_{f}}$ is plotted in Fig. 7 for fixed $G r=5, Q=0.8$ and $P r=0.71$. From the Fig. 7 , it is noted that for all $T a$ values $\overline{C_{f}}$ originally reduces and becomes asymptotically constant after reaching the highest value.

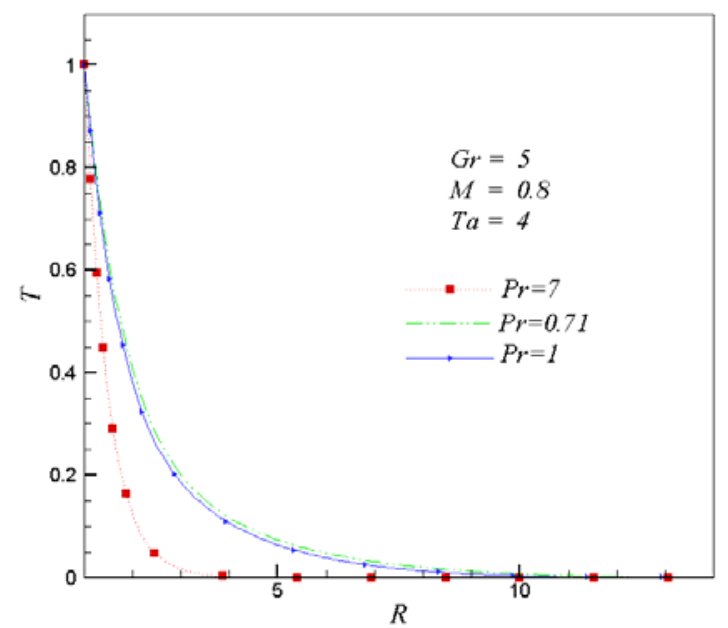

Fig. 5. Temperature profiles versus $R$.

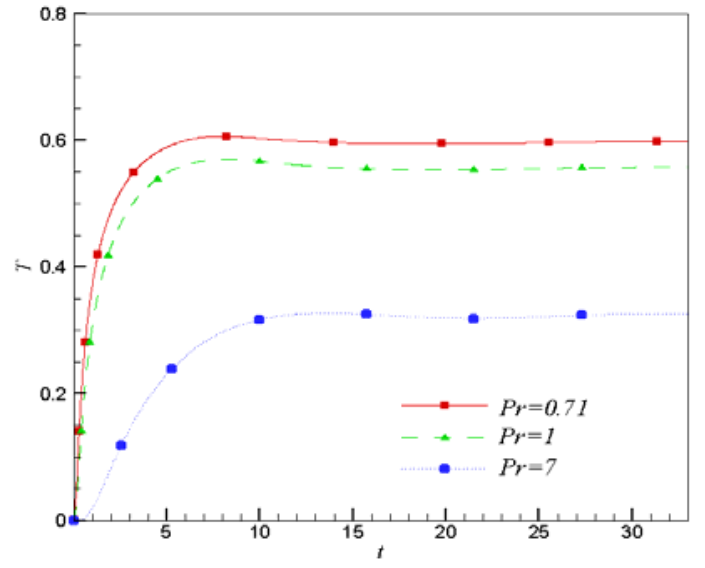

Fig. 6. Temperature profiles with respect to $t$.

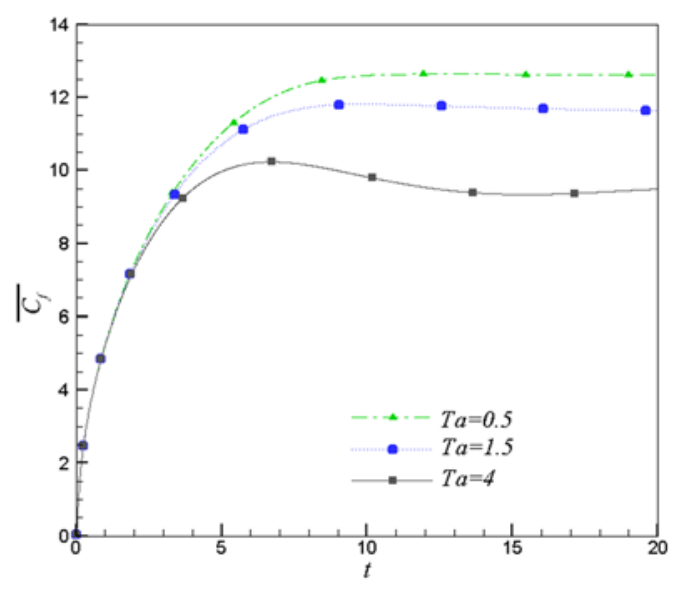

Fig. 7. Average skin-friction coefficient

The average heat transfer rate or Nusselt number is calculated from $\overline{N u}=-\int_{0}^{1}\left(\frac{\partial T}{\partial R}\right)_{R=1} d X$. In Fig. 8 the $P r$ effects on the simulated $\overline{N u}$ are shown for fixed values of other parameters such as $G r=5, T a=4$ and $Q=0.8$. As the value of $P r$ increases the values of $\overline{N u}$ increases. It is observed that initially, $\overline{N u}$ decreases but after a certain time it becomes time-independent because the effect of thermal diffusivity is increasing with time.

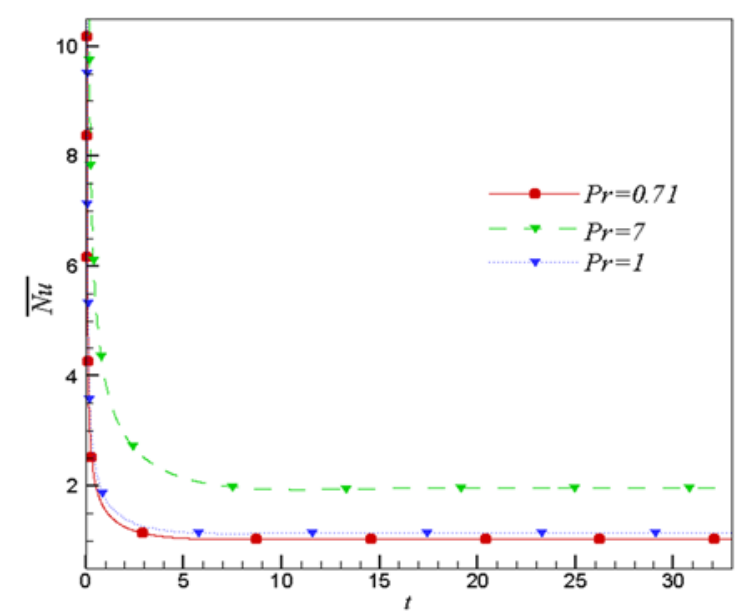

Fig. 8. Average Nusselt number 


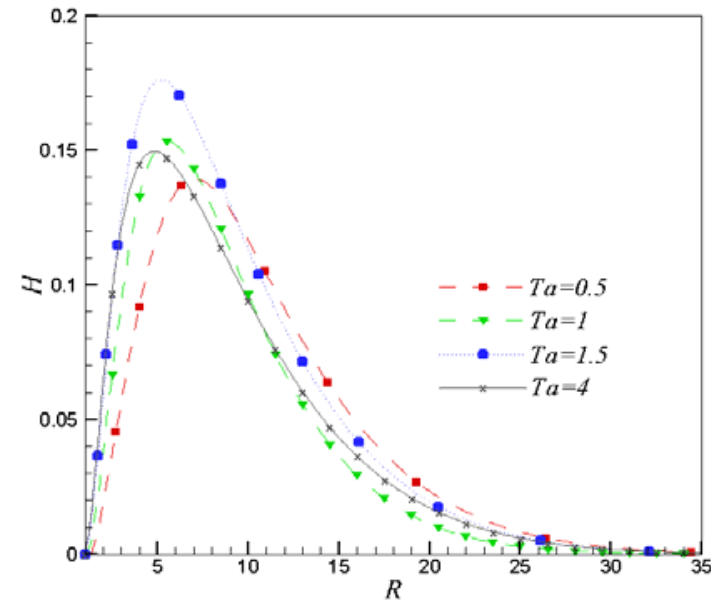

Fig. 9. Induced Magnetic field versus $R$

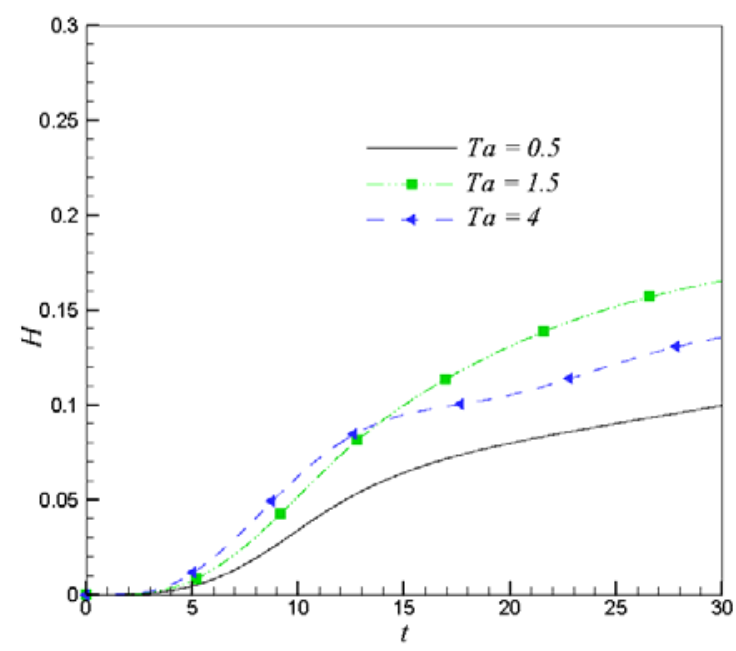

Fig.10. Induced Magnetic field versus $t$

From figure 9, magnetic field profiles can be seen to decline as $T a$ increases. In the upward direction, close the cylinder, the magnitude of the induced magnetic field reduces as $T a$ rises. Figure 10 demonstrates the magnetic field profiles versus time. It is noted that the magnetic field is also rising as time rises and reaches the stable state.

\subsection{Field Synergy Principle}

This principle defines if the angle between the temperature gradient and velocity vector is small then the heat transfer rate is high $[6,7]$. Figure 11 shows the angle between velocity and temperature gradient and which improves with $\mathrm{Ta}$. On the diagonal line shown in the Fig.11(i) and 11(iv), the field synergy values are calculated for different $\mathrm{Ta}$. The observation is that with growing $T a$, the rate of heat transfer is improved.

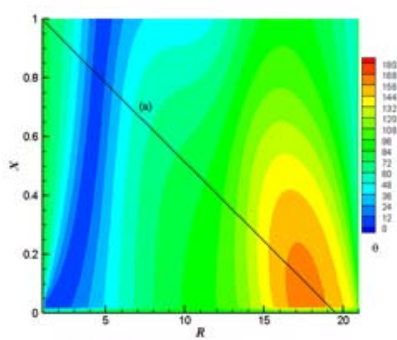

(i)

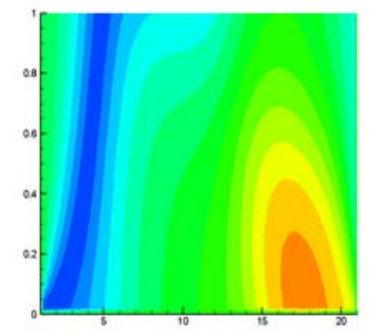

(iii)

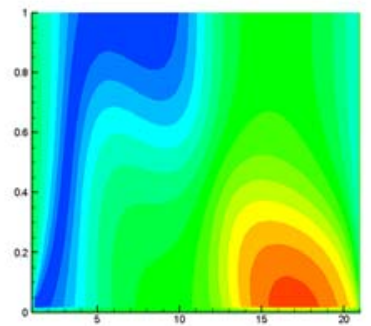

(ii)

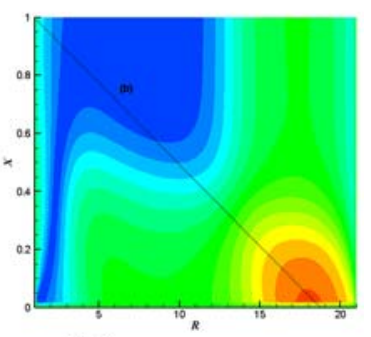

(iv)
Fig. 11. Field synergy variations for different $T a$ values with $G r=5, P r=0.71$ and $Q=0.8$.

\section{CONCLUSIONS}

In this paper, we have solved non-linear coupled partial differential equations numerically for the tangentially rotating MHD unsteady fluid flow past a semi-infinite vertical cylinder. For the increasing values of $T a$ and $Q$ the velocity, temperature and magnetic field effects are analyzed graphically. The velocity distribution decreases when $T a$ increases. When $T a$ increases the effect of Coriolis force increases. Similarly, the axial velocity decreased when $Q$ is increased. In both cases, the axial velocity decreases because the effect of kinematic viscosity decreases.

For fixed values of $P r=0.71, P m=0.1$ and $G r=5$, the temperature of the fluid rapidly decreased when $T a$ and $Q$ values increases. The magnetic field distribution rapidly changes when $Q$ increases.

When $T a$ and $Q$ increase the angle between temperature gradient and velocity vector is decreased, and hence the heat transfer rate is increased.

\section{NOMENCLATURE}

$x-$ axial coordinate

$X$ - non-dimensional axial coordinate

$r$ - radial coordinate

$r_{0}-$ radius of the cylinder

$R$ - non-dimensional radial coordinate

$t$ - time

$t^{\prime}-$ non-dimensional

$u$ - axial velocity

$v$ - transverse velocity

$T^{\prime}$ - temperature of the fluid

$B^{\prime}-$ induced magnetic field

$U-$ non-dimensional axial velocity

$V$ - non-dimensional transverse velocity

$T$ - non-dimensional temperature 
$H$ - non-dimensional induced magnetic field

$g-$ acceleration due to gravity

$\beta$ - coefficient of volume expansion

$B_{0}-$ constant magnetic induction

$\kappa-$ magnetic diffusivity

$\eta-$ thermal conductivity

$\omega-$ angular velocity

$\rho-$ density

$C_{p}$ - specific heat at constant pressure

$T_{\infty}^{\prime}-$ ambient temperature

$Q$ - Chandrasekhar number

Pr - Prandtl number

Pm - Magnetic Prandtl number

$\mathrm{Ta}$ - Taylor number

$\mathrm{Gr}$ - Grashof number.

\section{KEYWORDS}

Magnetohydrodynamics; Field synergy; Heat transfer, Rotation; Taylor number; Chandrasekhar number; numerical method.

\section{References}

1. E. M. Sparrow, J. L. Gregg, Laminar free convection heat transfer from the outer surface of a vertical circular cylinder, Trans. ASME., 78, 18231829 (1956).

2. S. Globe, Laminar steady-state magnetohydrodynamic flow in an annular channel, Physics Fluids, 2 404-407 (1959).

3. R. J. Goldstein, D. G. Briggs, Transient free convection about vertical plates and circular cylinders, Trans ASME C: J. Heat Transfer, 86, 490-500 (1964).

4. S. N. Dube, Magnetohydrodynamic flow between concentric rotating porous cylinders, Def. Sci. J., 21, 253-256 (1971).

5. Z. Guo, D. Li, B. Wang, A novel concept for convective heat transfer enhancement, Int. J. Heat Mass Trans., 41, 2221-2225 (1998).

6. Guo, Z., Tao, W., Shah, R. The field synergy (coordination) principle and its applications in enhancing single phase convective heat transfer. Int. J. Heat Mass Trans., 48, 1797- 1807 (2005).

7. Guo Z, Mechanism and control of convective heat transfer Coordination of velocity and heat flow fields. Chinese Sci Bull, 46, 596-599 (2001).

8. Ashish Paul, Flow past an accelerated horizontal cylinder in a rotating fluid, J. App. Fund. Sci., 2(2), 2395-5562 (2016). 